\title{
ASYMPTOTIC BEHAVIOR OF SOLUTIONS FOR A PARABOLIC EQUATION WITH NONLINEAR BOUNDARY CONDTTIONS
}

\author{
C. V. PAO
}

\begin{abstract}
This paper is concerned with the asymptotic behavior and nonexistence of global solutions for a linear parabolic equation under nonlinear boundary conditions. It is shown under certain conditions on the nonlinear boundary function that a global solution exists and converges to a steady-state solution while for another class of initial function the solution blows-up in finite time. In some special cases, threshold results for the convergence of the solution and its blowingup behavior are explicitly given.
\end{abstract}

I. Introduction. In an earlier paper [5] Walter established the existence and nonexistence of global solutions for the parabolic system

$$
\begin{gathered}
u_{t}-L u \equiv u_{t}-\left(\sum_{i, j=1}^{n} a_{i j}(x) u_{x_{i} x_{j}}+\sum_{i=1}^{n} b_{i}(x) u_{x_{i}}-c(x) u\right)=0 \\
\begin{array}{c}
\partial u / \partial \nu=f(x, u) \quad(t>0, x \in \Omega), \\
u(0, x)=u_{0}(x) \quad(x \in \Omega),
\end{array}
\end{gathered}
$$

in a framework of differential inequalities, where $\Omega$ is a bounded domain in $R^{n}$ with boundary $\partial \Omega, L$ is a uniformly parabolic operator in $\Omega, \partial / \partial \nu$ is the outward normal derivative on $\partial \Omega$ and $f$ is a continuous nonnegative function on $\partial \Omega \times$ $[0, \infty)$. The main conclusion in [5] states that if $f \equiv f(u)$ and if there exists $\eta_{0}>0$ such that $f, f^{\prime}$ are both positive and increasing for $u>\eta_{0}$, then global solutions exist when

$$
I \equiv \int_{\eta_{0}}^{\infty}\left[f(\eta) f^{\prime}(\eta)\right]^{-1} d \eta=\infty
$$

and the solution blows-up in finite time for a class of initial functions when $I<\infty$. This nonexistence problem due to a positive nonlinear function on the boundary surface has also been discussed by Levine and Payne [2] and by Pao [3] using a different argument. In all of the above papers the nonexistence of global solution requires a sufficiently large initial function $u_{0}$ in some sense. An interesting question along this line is whether global solution can exist when $I<\infty$, and, in case it does exist, whether it converges to a steady-state solution. With regard to the

Received by the editors August 2, 1979.

AMS (MOS) subject classifications (1970). Primary 35K60; Secondary 35B40, 35K20.

Key words and phrases. Asymptotic stability, nonexistence of global solutions, parabolic equations, nonlinear boundary conditions, upper and lower solutions. 
nonexistence problem it is desirable to know how large the initial function must be so that the corresponding solution blows-up in finite time. The purpose of this paper is to investigate these questions for the system (1.1)-(1.3) where $f \equiv f(x, u)$ may depend explicitly on $x$. It turns out that the answer to these questions depends on the relative magnitude between the functions $f$ and $c$ as well as the initial function $u_{0}$. This is to be expected from a physical point of view since $f$ represents a source on the boundary and the term $c u$ acts as a sink (for $c>0$ ) or a source (for $c<0$ ) in the interior domain. Our aim is to characterize the relative magnitude between the source and sink so that the existence or nonexistence of global solutions can be determined. In the special case of constant $c>0$ our characterization leads to a threshold result on the convergence (including existence) of a global solution to a steady-state solution for one class of initial functions and the blowing-up behavior of the solution for another class of initial functions. For example, in the one-dimensional domain $(0, l)$ with $L u=D u_{x x}-c_{0} u, f=b u^{1+\alpha}$, where $D, c_{0}, l, b, \alpha$ are positive constants, we show that a global solution exists and converges to zero when $u_{0}(x)<\rho_{0} \psi_{0}(x)$ and it blows-up in finite time when $u_{0}(x)>\rho^{*} \psi_{0}(x)$, where $\rho_{0}, \rho^{*}, \psi_{0}(x)>0$ are given explicitly in terms of the constants $D, c_{0}, l, b, \alpha$ (see equations (2.17) and (2.18)).

II. The main results. Throughout this paper we assume that the coefficients of $L$ and the boundary surface $\partial \Omega$ are sufficiently smooth and the matrix $\left(a_{i j}\right)$ is positive definite in $\bar{\Omega}$. The initial function $u_{0}$ and the boundary function $f$ are assumed nonnegative Hölder continuous in $\Omega$ and $\partial \Omega \times[0, \infty)$, respectively. For simplicity, we also assume that $f_{u}$ exists and is bounded on bounded subsets of $\bar{\Omega} \times R^{1}$. The above assumptions imply that the system (1.1)-(1.3) has at least one nonnegative local solution (cf. [4]).

In order to state the main results we need the following lemma which is our basis in establishing a connection among the functions $f, c$ and $u_{0}$.

LEMMA 1. Given any constant $\varepsilon<c(x)$ with $\varepsilon \not \equiv c(x)$ there exist positive functions $\psi(x)>0$ on $\bar{\Omega}$ and $\beta(x)>0$ on $\partial \Omega$ such that

$$
(L+\varepsilon) \psi=0 \text { in } \Omega \text { and } \partial \psi / \partial \nu-\beta(x) \psi=0 \text { on } \partial \Omega .
$$

The function $\psi$ can be normalized so that $\max \psi(x)=1$.

Proof. Consider the boundary-value problem

$$
(L+\varepsilon) \psi=0 \quad(x \in \Omega), \quad \partial \psi / \partial \nu=h(x) \quad(x \in \partial \Omega),
$$

where $h$ is a preassigned positive function on $\partial \Omega$. Since $c-\varepsilon>0$ and $c-\varepsilon \neq 0$ there exists a unique solution $\psi$ to $(2.2)$ (cf. [1, p. 137]). In view of $h>0$ on $\partial \Omega$ the maximum principle implies that $\psi(x)>0$ on $\bar{\Omega}$. The conclusion of the lemma follows by taking $\beta=h / \psi$.

In terms of the functions $\psi, \beta$ we now state

THEOREM 1. Let $c>0$ with $c \neq 0$ and let $\psi, \beta$ be the functions obtained in Lemma 1 for some $\varepsilon>0$ with $\varepsilon \neq c(x)$. If there exists a constant $\rho>0$ such that

$$
f(x, \eta)<\beta(x) \eta \quad \text { for } 0 \leqslant \eta<\rho, \quad(x \in \partial \Omega),
$$


then for any nonnegative $u_{0}<\rho \psi$ a global solution $u$ to the system (1.1)-(1.3) exists and satisfies the relation

$$
0<u(t, x)<\rho e^{-t} \psi(x) \quad(t>0, x \in \bar{\Omega}) .
$$

THeOREM 2. Let $\varepsilon^{*}<0$ such that $\varepsilon^{*}<c$ and $\varepsilon^{*} \neq c$, and let $\psi^{*}, \beta^{*}$ be the corresponding functions obtained in Lemma 1 with $\varepsilon=\varepsilon^{*}$. If there exists $\rho^{*}>0$ such that

$$
f\left(x, \eta \psi^{*}\right)>\beta^{*}(x) \eta \psi^{*} \text { for } \eta>\rho^{*} \quad(x \in \partial \Omega),
$$

then for any $u_{0}>\rho^{*} \psi^{*}$ the corresponding solution $u$ of (1.1)-(1.3) satisfies

$$
u(t, x) \geqslant \rho^{*} e^{\left(-e^{*}\right) t} \psi^{*}(x) \quad\left(t \in\left[0, T_{0}\right), x \in \bar{\Omega}\right)
$$

where $\left[0, T_{0}\right)$ is the maximum interval of existence of $u$. If, in addition, $f \equiv f(u)$ and $f$, $f^{\prime}$ are positive, increasing in $u \geqslant \eta_{0}$ for some $\eta_{0}>0$, then the solution $u$ grows to $\infty$ as $t \rightarrow \infty$ when $I=\infty$, and it blows-up in finite time when $I<\infty$.

COROllary. Let $c(x) \equiv c_{0}$ be a positive constant and let $\psi_{0}, \beta_{0}$ be the functions obtained in Lemma 1 with $\varepsilon=0$. If there exists a constant $\rho>0$ such that

$$
f(x, \eta)<\beta_{0}(x) \eta \quad \text { for } 0<\eta<\rho \quad(x \in \partial \Omega)
$$

then for $u_{0}<\rho \psi_{0}$ a global solution $u$ exists and converges to zero exponentially as $t \rightarrow \infty$. On the other hand, if there exists $\rho^{*}>0$ such that (2.7) is replaced by

$$
f\left(x, \eta \psi_{0}\right)>\beta_{0}(x) \eta \psi_{0} \text { for } \eta>\rho^{*} \quad(x \in \partial \Omega),
$$

then for $u_{0}>\rho^{*} \psi_{0}$ the solution grows to $\infty$ either in finite time or as $t \rightarrow \infty$.

The conclusion in Theorem 1 implies that if $c>0$ with $c \neq 0$ and if $f$ satisfies (2.3) then the global solution $u$ decays to zero exponentially as $t \rightarrow \infty$ when $\varepsilon>0$ and is uniformly bounded when $\varepsilon=0$. In terms of Lyapunov stability this implies that the zero steady-state solution is stable or asymptotically stable according to $\varepsilon=0$ or $\varepsilon>0$. In the latter case, a stability region is given by $\Lambda=\left\{u_{0}>0\right.$; $\left.u_{0}<\rho \psi\right\}$. On the other hand, if $f$ satisfies (2.5) with $\rho^{*}=0$ then the zero solution is unstable. This stability and instability property can be extended to nontrivial steady-state solution when either $f(x, 0) \neq 0$ or equation (1.1) is replaced by the inhomogeneous equation

$$
u_{t}-L u=q(x)
$$

(or both), where $q$ is a given continuous function. Here, as usual, a steady-state solution is a function $u_{s}(x)$ satisfying the boundary-value problem

$$
-L u=q \quad(x \in \Omega), \quad \partial \Omega / \partial \nu=f(x, u) \quad(x \in \partial \Omega) .
$$

The following theorems give some sufficient conditions for insuring the stability and instability of a given steady-state solution.

Theorem 3. Let $\psi, \beta$ be the functions obtained in Lemma 1 for some $0<\varepsilon<c$ with $\varepsilon \neq c(x)$, and let $u_{s}(x)$ be a steady-state solution of (2.10). If there exists $\rho>0$ such that

$$
\max _{|\eta|<\rho}\left[f_{u}\left(x, u_{s}(x)+\eta\right)\right]<\beta(x) \quad(x \in \partial \Omega),
$$


then a global solution $u$ to (1.1)-(1.3) exists and satisfies

$$
u_{s}(x)-\rho e^{-e t} \psi(x)<u(t, x) \leqslant u_{s}(x)+\rho e^{-e t} \psi(x) \quad(t>0, x \in \bar{\Omega})
$$

whenever $u_{s}-\rho \psi<u_{0}<u_{s}+\rho \psi$.

TheOREM 4. Let $\psi^{*}, \beta^{*}$ be the functions obtained in Lemma 1 for some $\varepsilon^{*}<0$ with $\varepsilon^{*} \neq c(x)$. If there exists $\rho^{*}>0$ such that

$$
\max _{\eta>\rho^{*}}\left[f_{u}\left(x, u_{s}(x)+\eta \psi^{*}(x)\right)\right]>\beta^{*}(x) \quad(x \in \partial \Omega),
$$

then the solution $u$ satisfies

$$
u(t, x)>u_{s}(x)+\rho^{*} e^{\left(-e^{*}\right) t} \psi^{*} \quad\left(t \in\left[0, T_{0}\right), x \in \partial \Omega\right)
$$

whenever $u_{0}>u_{s}+\rho^{*} \psi^{*}$, where $\left[0, T_{0}\right)$ is the maximum interval of existence of $u$.

REMARK. In Theorem 1 it is assumed that $c(x) \geqslant 0$ with $c(x) \neq 0$, and this assumption is not required in Theorem 2 . This requirement for the stability of a solution is a natural consequence of the system. Consider, for example, the special case $L u=\nabla \cdot(D \nabla u)-c u$, where $D \equiv D(x)>0$ is the diffusion coefficient. By integrating equation (1.1) over $\Omega$ and using the divergence theorem and the boundary condition (1.2) we obtain

$$
\frac{d}{d t} \int_{\Omega} u(t, x) d x=-\int_{\Omega} c(x) u(t, x) d x+\int_{\partial \Omega} D(x) f(x, u(t, x)) d x .
$$

The above relation demonstrates that if $c(x)<0$ (including $c \equiv 0$ ) the function $\bar{u}(t) \equiv \int_{\Omega} u(t, x) d x$ is an increasing function of $t$ whenever $f(x, u) \neq 0$ on $\partial \Omega$.

An example of bifurcation. When $c \equiv c_{0}>0$ is a constant, the corollary to Theorems 1 and 2 gives a threshold result on the stability and instability problem of the system. As a demonstration we consider the following simple one-dimensional model

$$
\left.\begin{array}{lll}
u_{t}-\left(D u_{x x}-c_{0} u\right)=0 & (t>0,0<x<l) \\
-u_{x}(t, 0)=b u^{1+\alpha}(t, 0), & u_{x}(t, l)=b u^{1+\alpha}(t, l) & (t>0) \\
u(0, x)=u_{0}(x) & (0<x<l)
\end{array}\right\}
$$

where $D, c_{0}, b, \alpha, l$ are positive constants. In this special problem the solution $\psi_{0}$ of (2.2) with $h=h_{0}>0$ and $\varepsilon=0$ is given by

$$
\psi_{0}(x)=A h_{0}\left[\left(e^{\mu x}+e^{-\mu x}\right)+\left(e^{\mu(l-x)}+e^{-\mu(l-x)}\right)\right] /\left[\mu\left(e^{\mu l}+e^{-\mu l}\right)\right]
$$

where $\mu=\left(c_{0} / D\right)^{1 / 2}$ and $A$ is a normalization factor so that $\max \psi_{0}(x)=1$. The corresponding function $\beta_{0}(x)$ is $\beta(0)=\beta(l)=\beta_{0}$ where

$$
\beta_{0}=\mu\left(e^{\mu l}-e^{-\mu l}\right) /\left[A\left(2+e^{\mu l}+e^{-\mu l}\right)\right] \text {. }
$$

The conditions on $f$ for stability and instability become, respectively,

$$
b \eta^{1+\alpha}<\beta_{0} \eta \text { for } 0<\eta<\rho, \quad b\left(\eta \psi_{0}\right)^{1+\alpha}>\beta_{0} \eta \psi_{0} \text { for } \eta>\rho^{*} .
$$

Choose $\rho=\rho_{0} \equiv\left(\beta_{0} / b\right)^{1 / \alpha}, \rho^{*}=\rho_{0} / \phi_{0}$, where $\phi_{0}=\min \phi_{0}(x)$. Then the requirement in (2.19) holds when $\eta<\rho_{0}$ and $\eta>\rho^{*}$, respectively. In view of the corollary we conclude that, for $u_{0}<\rho_{0} \phi_{0}$, a global solution to (2.16) exists and converges to 
zero, while for $u_{0}>\rho^{*} \phi_{0}$ the corresponding solution blows-up in finite time. The blowing-up behavior of the solution is due to the fact that the functions $f=b u^{1+\alpha}$ and $f^{\prime}=b(1+\alpha) u^{\alpha}$ are both increasing in $u$ for $u>0$ and the integral $I$ in (1.4) is finite.

III. Proof of the theorems. The proof of the above theorems is based on a comparison theorem established in [4] where the comparison functions are called upper and lower solutions (see also [6]). Here by an upper solution we mean a smooth function $\tilde{u}(t, x)$ satisfying the following inequalities.

$$
\left.\begin{array}{cl}
\tilde{u}_{t}-L \tilde{u}>0 & (t>0, x \in \Omega) \\
\partial \tilde{u} / \partial \nu>f(x, \tilde{u}) & (t>0, x \in \partial \Omega) \\
\tilde{u}(0, x)>u_{0}(x) & (x \in \Omega)
\end{array}\right\} .
$$

A lower solution is a smooth function $u(t, x)$ satisfying all the reversed inequalities in (3.1). In terms of upper and lower solutions we have the following theorem from [4].

THEOREM A. Let $\tilde{u}, \underset{\sim}{u}$ be a pair of upper and lower solutions such that $\underset{\sim}{\boldsymbol{u}}<\tilde{u}$ on $[0, T] \times \bar{\Omega}$ for every $\tilde{T}<\infty$ and let $f_{u}(x, u)$ exist and be bounded for $x \in \bar{\Omega}$, $\underset{\sim}{u}<u<\tilde{u}$. Then there exists a solution $u$ to (1.1)-(1.3) such that

$$
\underset{\sim}{u}(t, x)<u(t, x)<\tilde{u}(t, x) \quad(t>0, x \in \bar{\Omega}) .
$$

Proof of Theorem 1. It is clear from the hypothesis $u_{0}>0, f(x, 0)>0$ that the function $\underset{\sim}{\boldsymbol{u}}=\mathbf{0}$ satisfies all the reversed inequalities in (3.1). In view of Theorem $A$ it suffices to show that $\tilde{u}=\rho e^{-e t} \psi$ is an upper solution. Indeed, this is the case if

$$
\left.\begin{array}{cc}
\rho e^{-e t}(-\varepsilon \psi-L \psi)>0 & (t>0, x \in \Omega) \\
\rho e^{-e t} \partial \psi / \partial \nu>f\left(x, \rho e^{-e t} \psi\right) & (t>0, x \in \partial \Omega)
\end{array}\right\} .
$$

By the equation (2.1) in Lemma 1 the first relation is automatically satisfied while the second inequality is equivalent to

$$
\beta\left(\rho e^{-e t} \psi\right)>f\left(x, \rho e^{-e t} \psi\right) \quad(t>0, x \in \partial \Omega) .
$$

The above inequality is fulfilled by the condition (2.3) and the fact that $0<\rho \mathrm{e}^{-e} \psi$ $<\rho$. The result of Theorem 1 follows from Theorem $A$.

Proof of Theorem 2. Let $\underset{\sim}{u}=\rho^{*} e^{-e^{*} t} \psi^{*}$, where $\varepsilon^{*}<0$. Then $\underset{\sim}{u}$ is a lower solution if it satisfies the reversed inequalities in (3.3) when $\psi, \varepsilon$ are replaced by $\psi^{*}$, $\varepsilon^{*}$, respectively. Clearly these two inequalities follow from (2.1) and the fact that

$$
\beta^{*} \rho^{*} e^{-t} \psi^{*}<f\left(x, \rho^{*} e^{-e^{*}} \psi^{*}\right) \text { for } t>0 .
$$

In order to apply Theorem $A$, it is necessary to find an upper solution $\tilde{u}$ such that $\tilde{u}>\underset{\sim}{u}$. This is, in general, not possible since the solution may blow-up in finite time. To overcome this difficulty we use the approach of [4] by defining a suitable modified function $\tilde{f}(x, u)$ which is uniformly bounded on $\partial \Omega \times R^{+}$and coincides with $f(x, u)$ for $u$ in a preassigned finite interval $[0, N]$, where $R^{+}=[0, \infty)$. Using $u^{(0)}=\underset{\sim}{u}$ as an initial iteration we then construct a sequence $\left\{u^{(k)}\right\}$ from the linear 
system

$$
\left.\begin{array}{cl}
u_{i}^{(k)}-L u^{(k)}=0 & (t>0, x \in \Omega) \\
\partial u^{(k)} / \partial \nu+M u^{(k)}=M u^{(k-1)}+\tilde{f}\left(x, u^{(k-1)}\right) & (t>0, x \in \partial \Omega) \\
u^{(k)}(0, x)=u_{0}(x) & (x \in \Omega)
\end{array}\right\}_{k=1,2, \ldots,}
$$

where $M>\tilde{f}_{u}(x, u)$ on $\bar{\Omega} \times[0, N]$. It is easily seen from the property of a lower solution that the sequence $\left\{u^{(k)}\right\}$ is monotone nondecreasing (cf. [4]). By the uniformly bounded property of $\tilde{f}$, this sequence is bounded from above, and therefore it converges to a function $u^{*}>u$. A regularity argument shows that $u^{*}$ is a solution of the "modified problem," that is, the problem (1.1)-(1.3) with $f$ replaced by $\tilde{f}$. Since $\tilde{f}\left(x, u^{*}\right)=f\left(x, u^{*}\right)$ whenever the value of $u^{*}$ remains in $[0, N]$ and since $N$ can be chosen arbitrarily large we conclude that either $u^{*}$ coincides with the solution $u$ of the original problem or $u$ blows-up in finite time. In any case the relation (2.6) holds. Finally, if $f, f_{u}$ are positive increasing and the integral $I$ is finite then by the result of [5] the solution grows unbounded in a finite time provided that $u\left(t_{1}, x\right)$ is sufficiently large for some $t_{1}>0$. But this follows immediately from the relation (2.6). The proof of the theorem is completed.

Proof OF THE COROLlary. In view of (2.7) there exists $\delta>0$ such that $f(x, \eta)<$ $\left(\beta_{0}-\delta\right) \eta$ for $0<\eta<\rho, x \in \partial \Omega$. Since the functions $\psi, \beta$ obtained in Lemma 1 depend continuously on $\varepsilon$ there exists $\varepsilon_{1} \in\left(0, c_{0}\right)$ such that the corresponding functions $\psi_{1}, \beta_{1}$ in Lemma 1 (with $\varepsilon=\varepsilon_{1}$ ) satisfy $\beta_{1}>\beta_{0}-\delta, \psi_{1}>u_{0} / \rho$. This implies that $f(x, \eta)<\beta_{1} \eta$ for $0<\eta<\rho$ and $u_{0}<\rho \psi_{1}$. It follows from Theorem 1 that a global solution $u$ exists and satisfies $0<u(t, x)<\rho e^{-e_{1} t} \psi_{1}(x)$ which proves the first part of the corollary. Similarly, if (2.8) holds then there exists $\delta>0$ such that $f\left(x, \eta \psi_{0}\right) \geqslant\left(\beta_{0}+\delta\right) \eta \psi_{0}$ for $\eta \geqslant \rho^{*}$. The same argument as above shows that for some $\varepsilon^{*}<0$ the corresponding functions $\psi^{*}, \beta^{*}$ in Lemma 1 (with $\varepsilon=\varepsilon^{*}$ ) satisfy the relation $\beta^{*}<\beta_{0}+\delta, \psi^{*}<u_{0} / \rho^{*}$. The conclusion of the second part follows from Theorem 2 .

Proof of Theorem 3. In view of Theorem A it suffices to show that $\tilde{u}=u_{s}+$ $\rho e^{-e t} \psi$ and $\underset{\sim}{u}=u_{s}-\rho e^{-e t} \psi$ are upper and lower solutions, respectively. Indeed, since

$$
\left.\begin{array}{c}
\tilde{u}_{t}-L \tilde{u}=-L u_{s}+\rho e^{-e t}(-\varepsilon \psi-L \psi)=q \\
\partial \tilde{u} / \partial \nu=f\left(x, u_{s}\right)+\rho e^{-e t}(\beta \psi)
\end{array}\right\}
$$

$\tilde{u}$ is an upper solution if

$$
\beta\left(\rho e^{-e t} \psi\right)>f\left(x, u_{s}+\rho e^{-e t} \psi\right)-f\left(x, u_{s}\right)
$$

This relation follows from the hypothesis (2.11). The proof for the lower solution $\underline{\sim}$ is similar.

Proof of Theorem 4. To show the instability result (2.14) we observe from the relation (3.5) (with $\psi, \beta$ replaced by $\psi^{*}, \beta^{*}$ ) and the hypothesis (2.13) that $\underline{u}=u_{s}+\rho^{*} e^{-e^{*} t} \psi^{*}$ is a lower solution. The conclusion of the theorem follows from the same argument as in the proof of Theorem 2. 


\section{REFERENCES}

1. O. A. Ladyzenskaya and N. N. Uraltseva, Linear and quasilinear elliptic equations, Academic Press, New York, 1968.

2. H. A. Levine and L. E. Payne, Nonexistence theorem for the heat equation with nonlinear boundary conditions and for the porous medium equation backward in time, J. Differential Equations 16 (1974), 319-334.

3. C. V. Pao, Positive solution of a nonlinear boundary-value problem of parabolic type, J. Differential Equations 22 (1976), 145-163.

4. Asymptotic behavior and nonexistence of global solutions for a class of nonlinear boundary value problems of parabolic type, J. Math. Anal. Appl. 65 (1978), 616-637.

5. W. Walter, On existence and nonexistence in the lange of solutions of parabolic differential equations with a nonlinear boundary condition, SIAM J. Math. Anal. 6(1975), 85-90.

6. __ Differential and integral inequalities, Ergebnisse der Math. und ihrer Grenzgebiete, Band 55, Springer-Verlag, New York-Heidelberg-Berlin, 1970.

Department of Mathematics, North Carolna State Universtty, Raleigh, North Carolina 27650 